Supporting Information for

\title{
Self-Healing Thermoplastic Elastomer Formed from Triblock Copolymers with Dense 1,2,3-Triazole Blocks
}

Yanqiong Yang,${ }^{\S, \dagger}$ Yuri Kamon,$^{\S}$ Nathaniel A. Lynd,,${ }^{\star}$ and Akihito Hashidzume ${ }^{\S, *}$

$\S$ Department of Macromolecular Science, Graduate School of Science, Osaka University, 1-1

Machikaneyama-cho, Toyonaka, Osaka 560-0043, Japan

\# McKetta Department of Chemical Engineering, The University of Texas at Austin, Austin, Texas 78712-1589, United States 


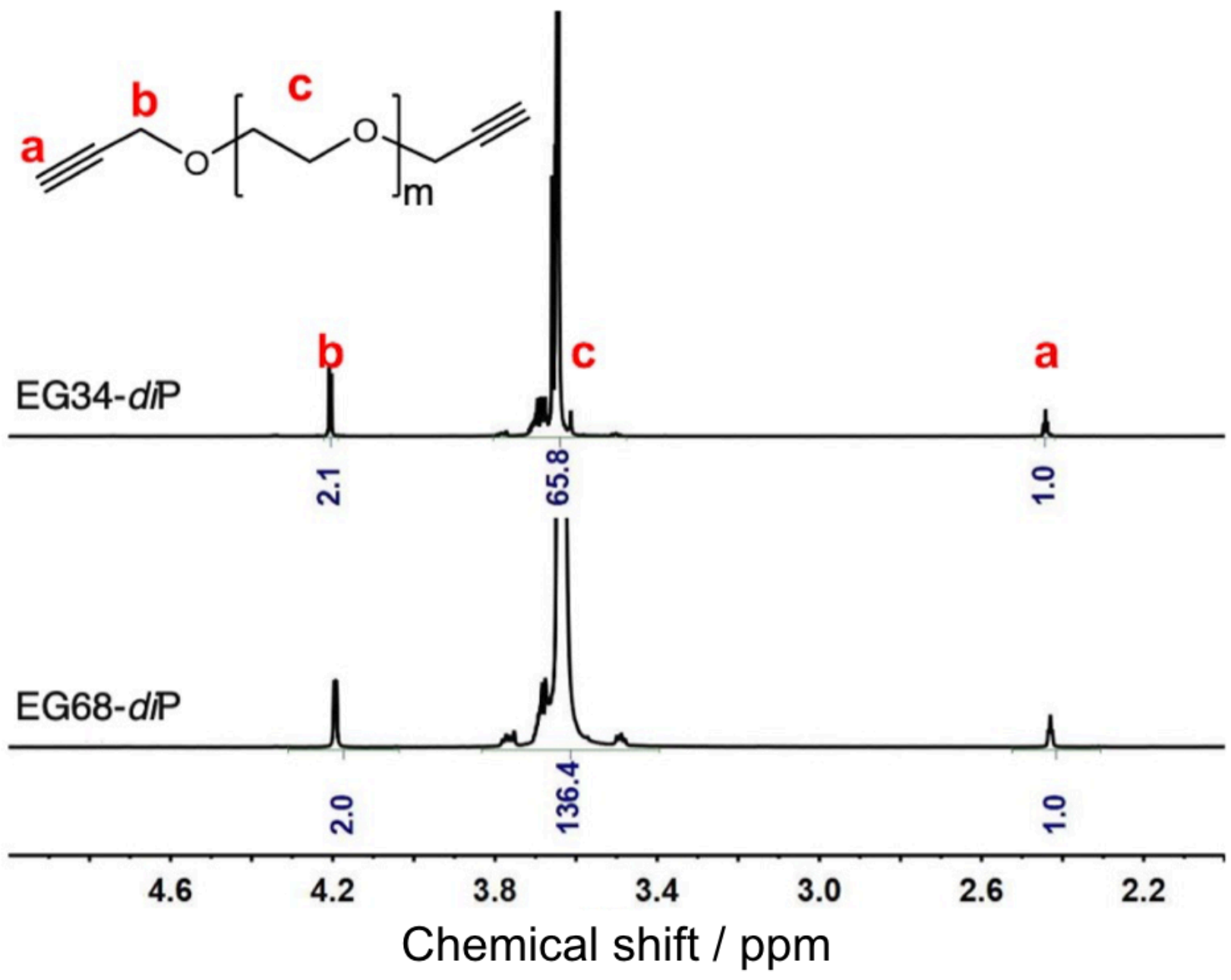

Figure S1. ${ }^{1} \mathrm{H}$ NMR spectra of EGm-diP in $\mathrm{CDCl}_{3}\left(500 \mathrm{MHz}, 25^{\circ} \mathrm{C}\right)$. 


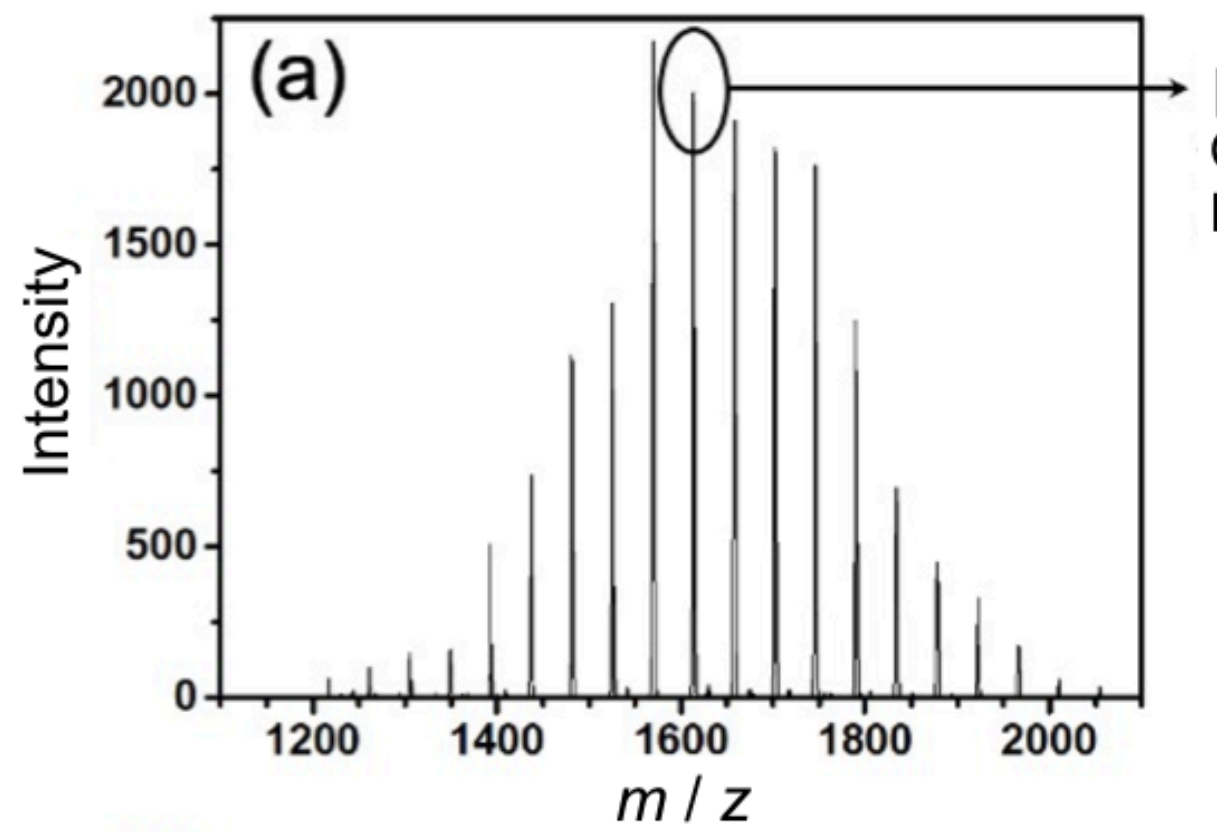

[EG34-diP + Na] ${ }^{+}$ Calcd: 1614.05

Found: 1614.10

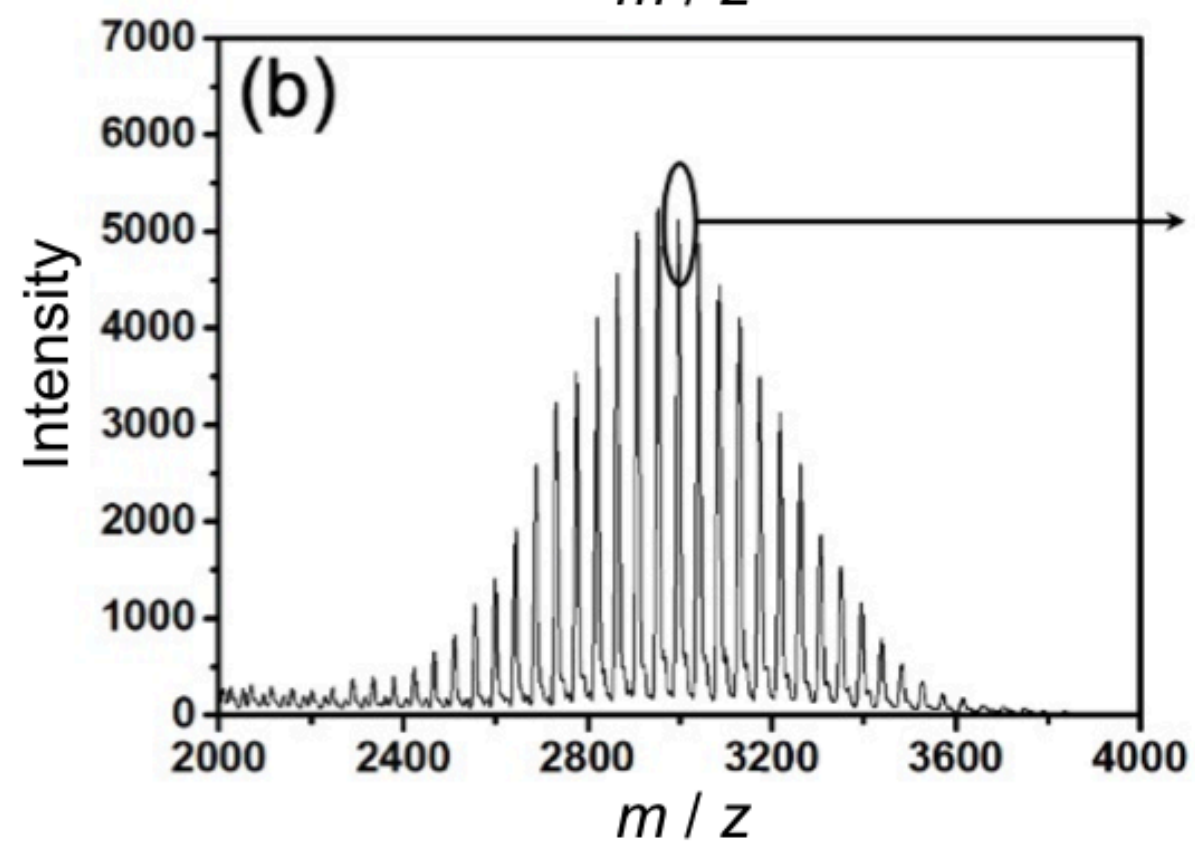

[EG68-diP + Na] ${ }^{+}$ Calcd: 2995.63 Found: 2995.70

Figure S2. MALDI-ToF-MS for EG34-diP(a) and EG68-diP (b). 


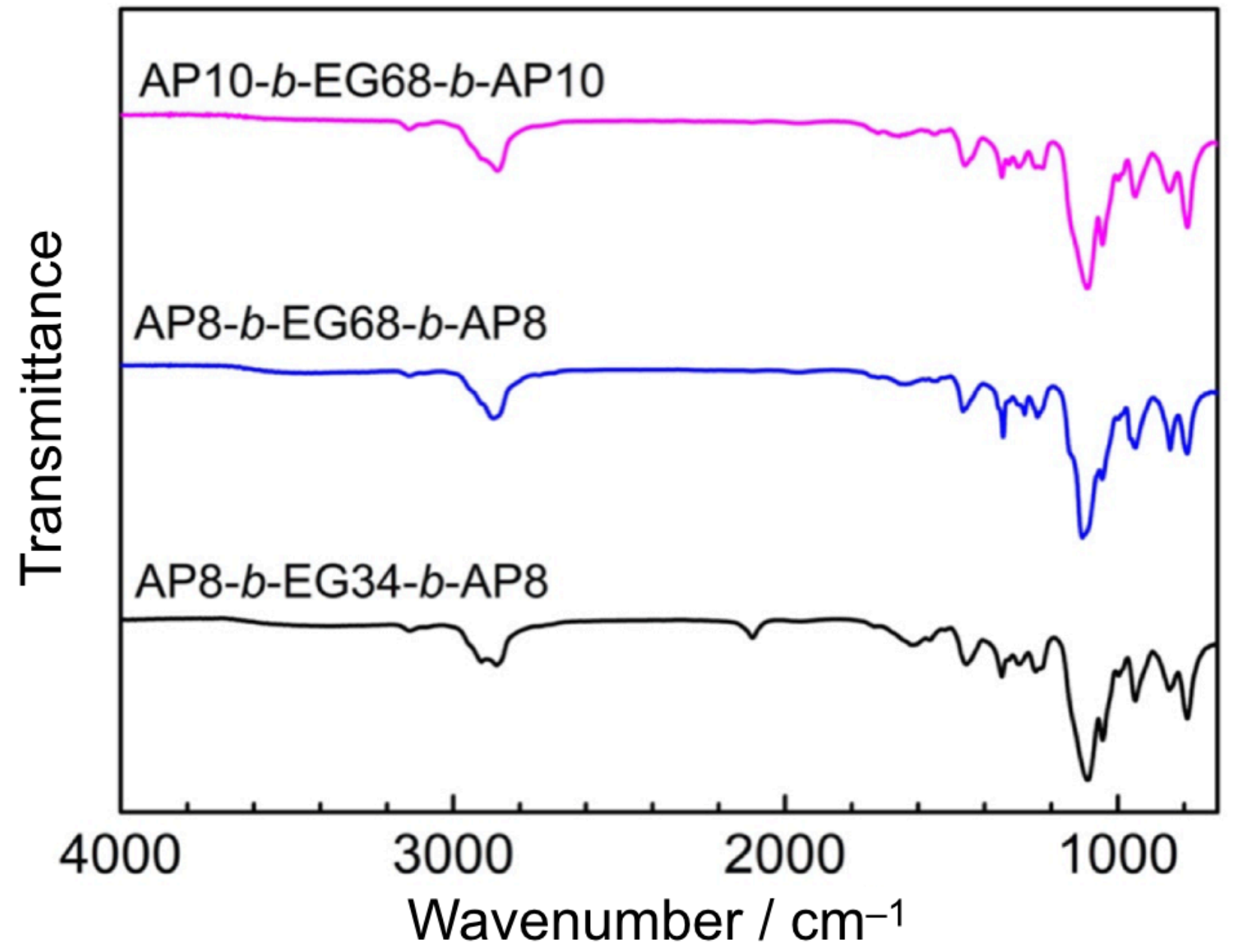

Figure S3. ATR-FTIR spectra for AP $n-b$-EGm- $b$-AP $n$ copolymers. 

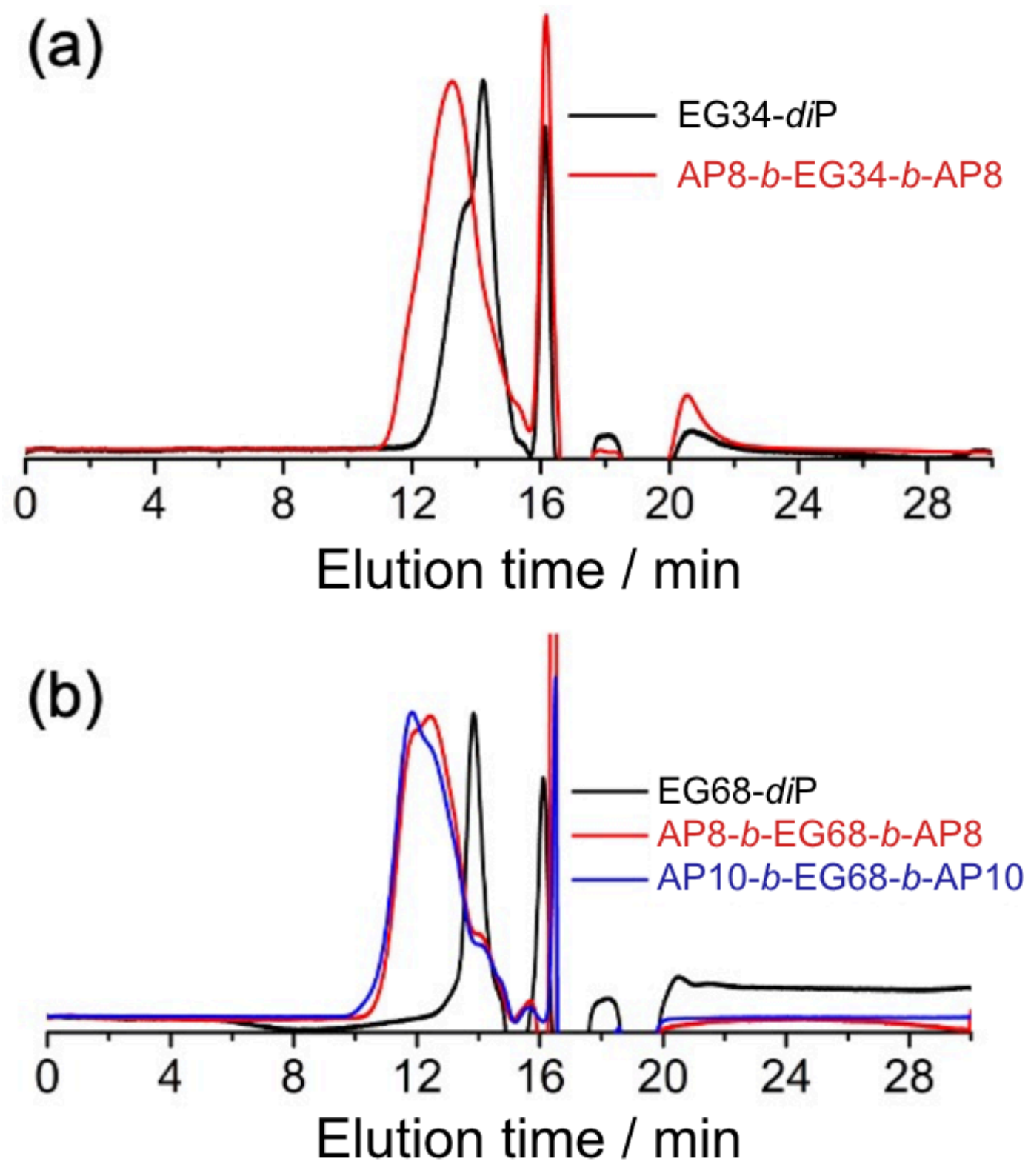

Figure S4. SEC data for EG34- $d i \mathrm{P}$ and AP8- $b$-EG34- $b$-AP8 (a), and EG68-diP, AP8- $b$-EG68- $b$ AP8, and AP10- $b$-EG68- $b$-AP10 (b). 


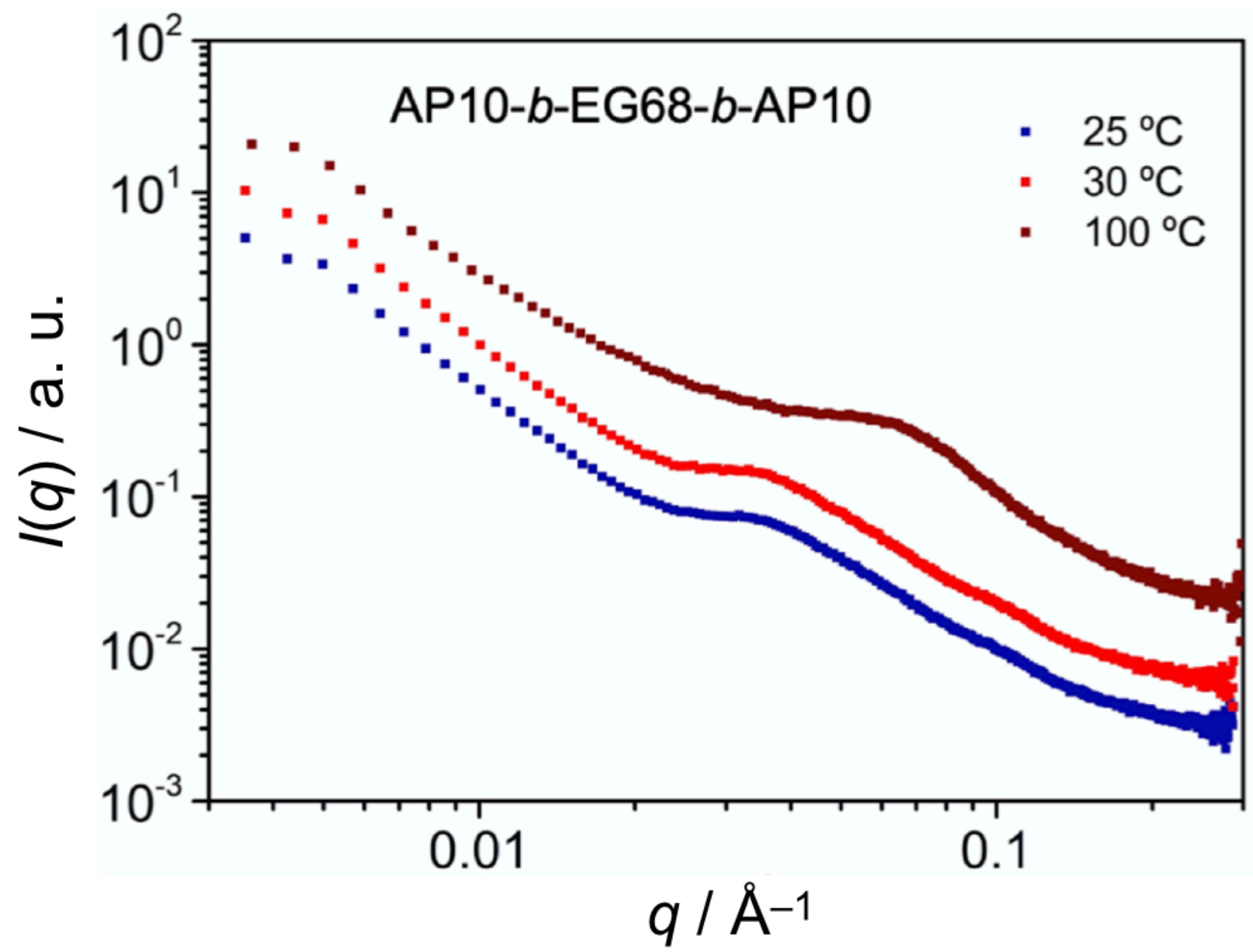

Figure S5. SAXS profiles of AP10- $b$-EG68- $b$-AP10 at different temperatures. 